\title{
Risk factors and outcome of urinary tract infections by extended spectrum beta lactamase producing microorganisms in children
}

\author{
*Shami Kumar RP1, Sai Lakshmi Ananya $\mathbf{T}^{2}$ \\ Sri Lanka Journal of Child Health, 2022; 51(1): 92-98 \\ DOI: http://dx.doi.org/10.4038/sljch.v51i1.10002
}

\begin{abstract}
Objectives: To describe the outcome and risk factors for Urinary Tract Infection (UTI) caused by Extended Spectrum Beta Lactamase (ESBL) producing microorganisms, in children aged 2 months to 15 years, at a tertiary care centre in South India over a period of 1 year.
\end{abstract}

Method: During the study period, there were 352 children with UTI, of which 100 had ESBL UTI and they were included in the study. These 100 children were analysed for risk factors of UTI, nature of treatment, duration of hospital stay and response to treatment and these were recorded in the preformed proforma. Urine routine, urine culture and sensitivity, ultrasonogram, micturating cystourethrogram and dimercapto-succinic acid (DMSA) scan were done for all patients. If colony count was $\geq 10^{5}$ colony forming units (CFUs) $/ \mathrm{ml}$ on clean catch sample or urobag sample or any number in catheterized or suprapubic sample with significant ESBL strain growth on urine culture were recorded along with sensitivity pattern. Follow up was done for 3 months.

Results: Age and sex distribution of ESBL UTI were $20(37 \%)$ boys and $13(28.2)$ girls in the age group 2 months to 2 years, $15(27.7 \%)$ boys and 15 $(32.6 \%)$ girls in the age group 2 to 5 years, 12 $(22.2 \%)$ boys and $5(10.8 \%)$ girls in the age group 5 to 10 years and $7(11.9 \%)$ boys and $13(28.2 \%)$ girls in the age group 10 to 15 years. Common risk factors for ESBL UTI in our study were recent antibiotic use within the last 3 months (55\%),

\footnotetext{
${ }^{1}$ Saveetha Medical College and Hospital, Chennai, Tamil Nadu, India, ${ }^{2}$ Rich Hospitals, Nellore, Andhra Pradesh, India

*Correspondence: drshamiich@gmail.com$$
\text { iD }
$$$$
\text { https//orcid.org/ 0000-0002-4424-0976 }
$$

(Received on 04 May 2021: Accepted after revision on 18 June 2021)

The authors declare that there are no conflicts of interest

Personal funding was used for the project.

Open Access Article published under the Creative

Commons Attribution CC-BY (c) (i) License
}

previous UTI (40\%), underlying renal problems $(31 \%)$, recent hospitalization within the last 3 months $(26 \%)$, antibiotic prophylaxis $(24 \%)$, recent catheterization within the last 3 months $(18 \%)$ and comorbidities $(8 \%)$. Seventy patients were followed up after treatment for a period of 3 months. Among them $14(20 \%)$ had another episode of UTI.

Conclusions: ESBL UTI was common in the age group 2 months to 2 years and was more common in males. Fever was the most common presentation. Underlying renal problems, recent antibiotic use, recent hospitalization, recent catheterization, previous UTI and antibiotic prophylaxis were found to be significant risk factors for ESBL UTI. Higher rates of pyelonephritis, voiding dysfunction, renal abnormalities and VUR were detected in ultrasonogram of patients with ESBL UTI. VUR was more common in patients with ESBL UTI. Renal scarring was more frequent in patients with ESBL UTI. Recurrence of UTI was more likely in patients with ESBL UTI.

(Key words: UTI, ESBL, Risk factor, Recurrence)

\section{Introduction}

Urinary tract infection (UTI) is one of the predominant causes of febrile illness in children with demanding antimicrobial therapy and it is also a common nosocomial infection ${ }^{1,2}$. Overall, $8 \%$ of females and $2 \%$ of males, experience at least one incident of UTI by seven years of age, and recurrence occurs in up to $30 \%$ during a year ${ }^{1}$. In infancy, UTI has a male to female ratio of 3-5:1. Beyond infancy, male to female ratio is $1: 10^{3}$. Worldwide prevalence of Extended Spectrum Beta Lactamase (ESBL) infections has been steadily increasing with predominantly Escherichia coli and Klebsiella uropathogens and they were described as a pandemic in most regions ${ }^{1}$. ESBLs were first described in $1983^{4}$. Beta lactamases produced by bacteria protect against lethal effects of penicillins, cephalosporins and monobactams on their cell wall synthesis. ESBLs have now become widespread all over the world ${ }^{4}$. UTI due to ESBL range from uncomplicated infection to lifethreatening sepsis. Furthermore, therapy of ESBL UTI is more difficult as these strains are increasingly resistant to other non-Beta lactam antibiotics, thus 
promoting increased usage of expensive broad spectrum antibiotics like carbapenems ${ }^{5}$.

Infancy, delay in starting treatment, recurrences and vesico-ureteric reflux (VUR) are risk factors for renal scarring $6,7,8$. South India ESBL UTI was reported in $40 \%$ of the population and among this Escherichia coli and Klebsiella were $46.3 \%$ and $25 \%$, respectively ${ }^{7}$. Preventing the spread of ESBLproducing Enterobacteriaceae in the community and healthcare settings is a significant global challenge with the increasing trend of antimicrobial resistance, which is even more significant in the sub-Saharan African region. Furthermore, high levels of antimicrobial resistance related to ESBL producing Enterobacteriaceae complicate individual patient care, increasing the mortality and morbidity associated with common infectious diseases in children like UTI ${ }^{9}$. ESBL infections in children are associated with longer hospital stays, frequent complications, and increased mortality at higher than adult rates in certain regions ${ }^{6}$. Infancy, children on uroprophylaxis, recent antibiotic usage, recurrent urinary tract infections (UTI), urinary tract anomalies, non-renal comorbidities and male gender should guide antibiotic therapy pending culture results in ESBL UTI children ${ }^{6,7,8}$. Fastidious hygiene, patient isolation, cohorting, dedicated staff and alternating antibiotic regimen policies were all employed to control outbreaks of ESBL infection with varying degrees of success. The drug with preserved efficacy for ESBL producers and nonESBL producers is nitrofurantoin ${ }^{6}$.

National and international programmes dedicated to the health of children worldwide need to consider the emerging threat of ESBL-producing bacteria in both resource-rich and resource-challenged countries, and research efforts should focus on the molecular characterization of ESBL types as well as additional controlled studies assessing risk factors and outcomes in children ${ }^{10}$. Many studies have been carried out in various centres globally to assess the risk factors of infections associated with ESBL producing strains in adults. However, studies in this regard on Indian children, especially from the South are limited. Hence this study was undertaken.

\section{Objectives}

To describe the outcome and risk factors for UTI caused by ESBL producing microorganisms in children aged 2 months to 15 years.

\section{Method}

A prospective observational study was carried out in Rich Hospitals, Nellore, Andhra Pradesh, a tertiary care centre in South India, over a period of 1 year.
Inclusion criteria: Children aged 2 months to 15 years with significant ESBL strain growth on urine culture and sensitivity $\left(>10^{5} \mathrm{CFUs} / \mathrm{ml}\right)$ on clean catch sample or any colony count on suprapubic aspiration / catheterized sample.

Exclusion criteria: ESBL positivity on urine culture and sensitivity without clinical manifestations of UTI (asymptomatic bacteriuria).

The study population consisted of 100 consecutive children admitted to the hospital due to communityacquired UTI (ESBL UTI). Child's history, clinical examination and risk factors for UTI were recorded in the proforma. Urine routine and urine culture and sensitivity were done for all patients. If the colony count was $\geq 10^{5}$ CFUs $/ \mathrm{ml}$ on clean catch sample or urobag sample or any number in catheterized or suprapubic sample then the organism and sensitivity pattern was recorded. Of the total 352 children, in 326 midstream urine or catheter sample was collected and in 26 children urine specimens were collected with urine collecting bag due to unavoidable circumstances, out of which 9 were reported with ESBL UTI; these ESBL UTI positive children again underwent catheterised urine sample culture for confirmation; the other 17 children were reported as non-ESBL UTI.

Urine cultures were repeated when contamination was suspected, e.g., mixed growth of two or more pathogens, or growth of organisms that normally constitute the peri-urethral flora (lactobacilli in healthy girls and enterococci in infants and toddlers). The culture was also repeated in situations where UTI was strongly suspected but colony counts were equivocal as per microbiologist's suggestion.

Contamination by peri-urethral and preputial organisms was minimized by washing the genitalia with soap and water. Antiseptic washes and forced preputial retraction were not advised. The specimen was directly collected in a sterile glass or plastic bottle. In infants, urine samples were preferably obtained by urethral catheterization. Prompt plating of the urine specimen was done within one hour of collection and if delay was anticipated, the sample was stored in a refrigerator.

Ultrasonogram, micturating cystourethrogram (MCU) and dimercapto-succinic acid (DMSA) scan were done as per Indian Academy of Pediatrics (IAP)/American Academy of Pediatrics (AAP) Guidelines ${ }^{11}$. Positive findings of ultrasonogram, Kidney Ureters Bladder (KUB) like pyelonephritis, cystitis, faecal loading, renal abnormalities, postvoid significant residual urine volume and VUR were noted. Grade 1 to grade 5 VUR or any 
anatomical abnormality in bladder and urethra were considered as abnormal MCU in patients who underwent MCU. Renal scarring or inflammation either unilateral or bilateral was considered as abnormal DMSA in patients who underwent DMSA. Nature of treatment, duration of hospital stay and response to treatment were recorded in case of inpatients. In case of outpatients, duration and response to treatment was recorded. Follow up was done for 3 months. Follow up details (UTI recurrence, uroprophylaxis) were recorded.

Ethical issues: Approval for the study was obtained from the Institutional Ethics Committee of Rich Hospitals, Nellore, Andhra Pradesh, India (No. RICH/RES/2020/048). Written Informed consent was obtained from the parents of the children included in the study.

Statistical analysis: Data were entered in MSExcel spreadsheet, validated and analysed using SPSS version 11. Incidence was calculated from data. Chi square test and Fischer $t$ test were used to analyse variables.

\section{Results}

During the study period, there were 352 children with UTI, of whom 100 had ESBL UTI and were enrolled into the study group. Hence total percentage of ESBL UTI was $100 / 352 \times 100=28.4 \%$. The study flow chart is shown in Figure 1.

Table 1 shows the age and sex distribution of ESBL.

Table 2 shows the clinical features of ESBL UTI.

Table 3 shows the risk factors of ESBL.

Table 4 shows the investigations used in the study.

Table 5 is the logistic regression analysis of risk factors for UTI.

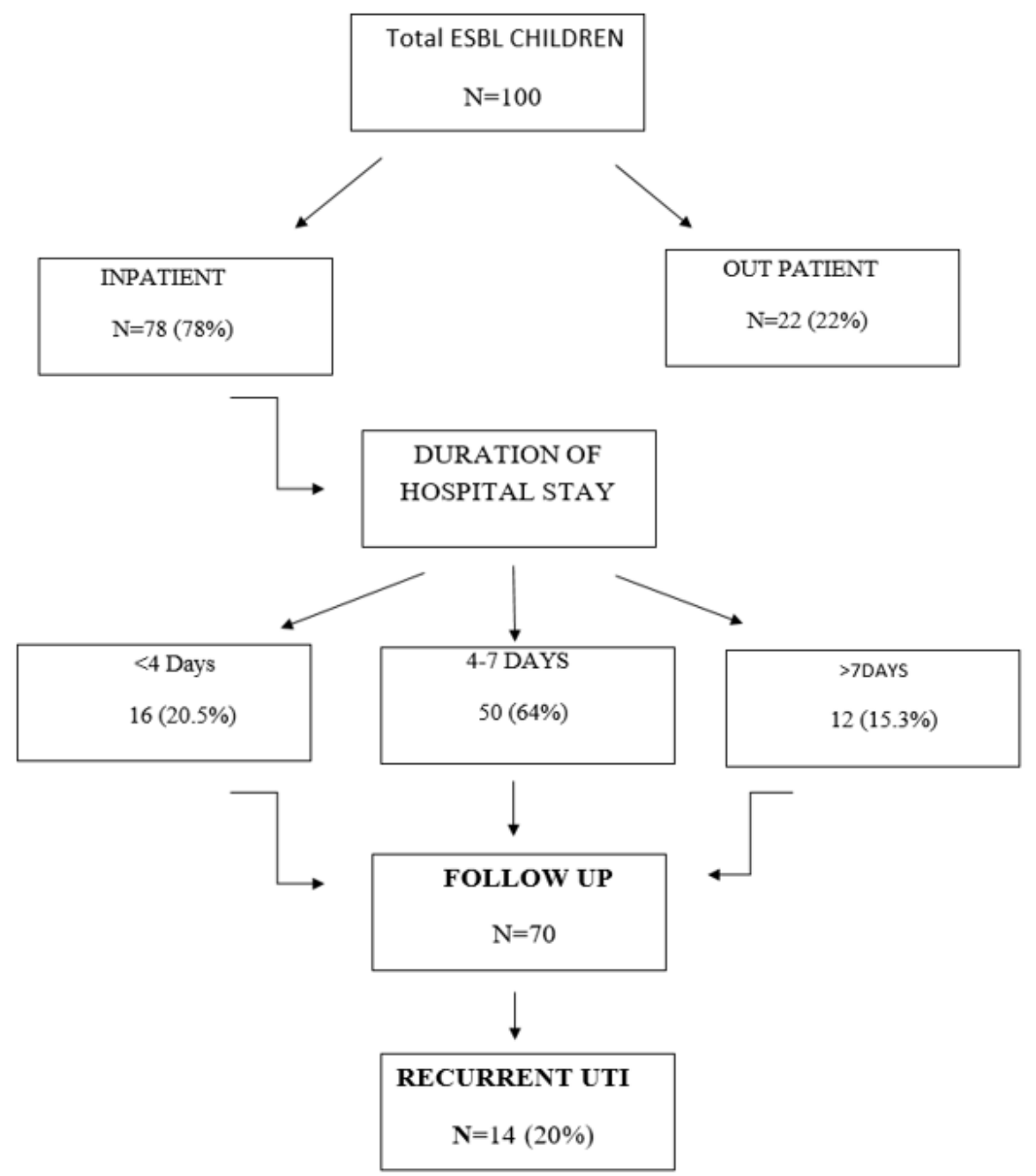

Figure 1: Study flowchart 
Table 1: Age and sex distribution of Extended Spectrum Beta Lactamase (ESBL)

\begin{tabular}{|c|c|c|c|c|}
\hline Age group & $\begin{array}{c}\text { ESBL }(n=100) \\
n(\%)\end{array}$ & $\begin{array}{c}\text { Non ESBL }(\mathrm{n}=\mathbf{2 5 2}) \\
\text { n (\%) }\end{array}$ & Odds ratio & p-value \\
\hline 2 months $-<2$ years & $33(33)$ & $45(17.9)$ & 1.97 & $0.01 *$ \\
\hline 2 years $-<5$ years & $30(30)$ & $70(27.8)$ & 1.22 & 0.4 \\
\hline 5 years $-<10$ years & $17(17)$ & $82(32.5)$ & 0.46 & 0.04 \\
\hline 10 years -15 years & $20(20)$ & $55(21.8)$ & 0.8 & 0.76 \\
\hline Sex & & & & \\
\hline Male & $54(54)$ & $139(55.2)$ & \multirow[t]{2}{*}{0.95} & \multirow[t]{2}{*}{0.84} \\
\hline Female & $46(46)$ & $113(44.8)$ & & \\
\hline
\end{tabular}

Table 2: Symptomatology of patients with ESBL UTI $(n=100)$

\begin{tabular}{|l|c|}
\hline \multicolumn{1}{|c|}{ Symptom } & n (\%) \\
\hline Fever & $78(78)$ \\
\hline Urinary frequency & $37(37)$ \\
\hline Dysuria & $25(25)$ \\
\hline Poor feeding & $07(07)$ \\
\hline Vomiting & $15(15)$ \\
\hline Loose stools & $06(06)$ \\
\hline Pain in abdomen & $26(26)$ \\
\hline Chills and rigors & $17(17)$ \\
\hline Enuresis & $19(19)$ \\
\hline
\end{tabular}

Table 3: Risk factors of Extended Spectrum Beta Lactamase (ESBL)

\begin{tabular}{|l|c|c|c|c|}
\hline \multicolumn{1}{|c|}{ Risk factor } & $\begin{array}{c}\text { ESBL (n=100) } \\
\mathbf{n}(\mathbf{\%})\end{array}$ & $\begin{array}{c}\text { Non ESBL (n=252) } \\
\mathbf{n}(\mathbf{\%})\end{array}$ & Odds ratio & p-value \\
\hline Renal problems & $31(31)$ & $210(8.3)$ & 4.942 & $0.000^{*}$ \\
\hline Recent antibiotic use & $55(55)$ & $38(15.1)$ & 6.883 & $0.000^{*}$ \\
\hline Recent hospitalisation & $26(26)$ & $21(08.3)$ & 3.865 & $0.000^{*}$ \\
\hline Previous urinary tract infection & $40(40)$ & $30(11.9)$ & 4.933 & $0.000^{*}$ \\
\hline Recent catheterisation & $18(18)$ & $06(02.4)$ & 3.238 & $0.001^{*}$ \\
\hline Neurological abnormality & $04(04)$ & $07(02.8)$ & 1.458 & 0.515 \\
\hline Constipation & $12(12)$ & $37(14.7)$ & 0.792 & 0.512 \\
\hline Comorbidities & $08(08)$ & $01(0.4)$ & 21.826 & $0.000^{*}$ \\
\hline
\end{tabular}

Table 4: Investigations used in the study

\begin{tabular}{|c|c|c|}
\hline Investigation & & $\begin{array}{c}\text { ESBL } \\
\text { Number (\%) }\end{array}$ \\
\hline \multirow{6}{*}{$\begin{array}{l}\text { Ultrasonography } \\
\quad(n=100)\end{array}$} & Pyelonephritis & $36(36.0)$ \\
\hline & Cystitis & $34(34.0)$ \\
\hline & Post void significant residual bladder volume & $10(10.0)$ \\
\hline & Faecal loading & $08(08.0)$ \\
\hline & Renal abnormality & $12(12.0)$ \\
\hline & Vesico ureteric reflux (VUR) & $10(10.0)$ \\
\hline $\begin{array}{c}\text { Micturating cystourethrogram } \\
(n=27)\end{array}$ & $\begin{array}{l}\text { Grade 1-5 VUR / abnormality in bladder and } \\
\text { urethra }\end{array}$ & $15(55.5)$ \\
\hline $\begin{array}{c}\text { Dimercaptosuccinic acid scan } \\
(n=42)\end{array}$ & $\begin{array}{l}\text { Renal parenchymal inflammation and renal } \\
\text { scarring (unilateral/bilateral) }\end{array}$ & $32(76.1)$ \\
\hline \multirow{3}{*}{$\begin{array}{l}\text { Urine culture } \\
(n=100)\end{array}$} & Escherichia coli & $81(81.0)$ \\
\hline & Klebsiella & $16(16.0)$ \\
\hline & Enterobacter & $02(03.0)$ \\
\hline
\end{tabular}


Table 5: Logistic regression analysis of risk factors for urinary tract infection

\begin{tabular}{|l|c|c|c|c|c|c|c|}
\hline \multirow{2}{*}{ Complication } & B & SE & t score & df & \multirow{2}{*}{$\mathbf{p}$} & \multicolumn{2}{c|}{$\mathbf{9 5 \%}$ CI } \\
\cline { 4 - 8 } & & & & & & Lower & Upper \\
\hline Known renal problems & 0.796 & 0.033 & 23.972 & 1 & $0.000^{*}$ & 0.731 & 0.861 \\
\hline Recent hospitalization & 0.723 & 0.065 & 11.058 & 1 & $0.000^{*}$ & 0.594 & 0.852 \\
\hline Recent catheterization & 0.457 & 0.099 & 4.631 & 1 & $0.000^{*}$ & 0.263 & 0.652 \\
\hline Recent antibiotic use & 0.211 & 0.066 & 3.205 & 1 & $0.001^{*}$ & 0.082 & 0.341 \\
\hline Previous urinary tract infection & 0.145 & 0.063 & 2.302 & 1 & $0.022^{*}$ & 0.021 & 0.268 \\
\hline Neurological abnormality & -0.037 & 0.089 & -0.416 & 1 & 0.678 & -0.212 & 0.138 \\
\hline Constipation & -0.131 & 0.079 & -1.651 & 1 & 0.100 & -0.287 & 0.025 \\
\hline Comorbidities & 0.678 & 0.065 & 10.436 & 1 & $0.000^{*}$ & 0.550 & 0.805 \\
\hline
\end{tabular}

B: Coefficient for constant, SE: Standard error, df: degrees of freedom for Wald Chi square test, CI: confidence interval

\section{Discussion}

This prospective hospital-based observational study of ESBL UTI in children between 2 months to 15 years, enrolled a total of 100 children as per pre-defined inclusion criteria. The incidence of ESBL UTI in children varies worldwide and ranges from $10.4 \%-45.1 \%{ }^{12-18}$, whereas in this study it was $28.4 \%$. This was less than the incidence reported from Aligarh, India by Mohammed A, et a ${ }^{12}$ and Delhi, India by Kaur N, et al ${ }^{13}$ which were $45.1 \%$ and $42 \%$ respectively. Children between 2 months to 2 years had higher rates of ESBL UTI (33\%) than other age groups and this age group had statistically significant higher rates of ESBL UTI [Table 1]. This was similar to the study by Kizilca $\mathrm{O}$, et $a l^{5}$ where age less than one year had higher rates of ESBL UTI. On analysis of ESBL UTI, we found that $78 \%$ of children presented with fever, similar to the study by Topalogulu R, et $a l^{19}$ who found that fever was most common presenting symptom in patients with ESBL UTI (40.6\%) [Table 2].

The most common risk factor for ESBL UTI in this study was recent antibiotic use within the last 3 months which was seen in $55 \%$ children [Table 3]. Other risk factors for ESBL UTI in this study in descending order were previous UTI (40\%), underlying renal problems (31\%), hospitalization within the last 3 months $(26 \%)$, antibiotic prophylaxis $(24 \%)$, catheterization within the last 3 months (18\%) and co-morbidities (8\%). Our findings were comparable to previous studies by Topalogulu $\mathrm{R}$, et $a l^{19}$ and Dayan $\mathrm{N}$, et $a l^{20}$. In the study by Topalogulu R, et $a l^{19}$, the most common risk factor for ESBL UTI was recent antibiotic use within the last 3 months (79\%) followed by renal problems (63.9\%), previous UTI (54.8\%) and recent hospitalization within the last 3 months (47\%). In the study by Dayan N, et $a l^{20}, 40 \%$ of children with previous UTI, $32 \%$ of children with underlying renal problems and $32 \%$ of children on antibiotic prophylaxis had ESBL UTI. Fan N, et $a l^{21}$ demonstrated that risk factors for ESBL UTI were similar to our study except for previous UTI, which was not found as a risk factor. Constipation and underlying neurological disease were found as risk factors for ESBL UTI in our study, whereas Fan N, et $a l^{21}$ found neurological disease to be a significant risk factor for ESBL UTI.

In patients with ESBL UTI, ultrasonogram showed pyelonephritis in $36 \%$, cystitis in $34 \%$, renal abnormalities in $12 \%$, VUR in $10 \%$ and post void significant residual urine volume in $10 \%$ [Table 4], whereas the study by Dayan $\mathrm{N}$, et al ${ }^{20}$ found cystitis in $24 \%$ and pyelonephritis in $76 \%$ of patients with ESBL UTI. In our study 15/27 (55.5\%) of children with ESBL UTI had abnormal MCU, which was comparable to studies by Topalogulu $\mathrm{R}$, et $a l^{19}$ and Dotis $\mathrm{J}$, et $a l^{4}$ which reported VUR in $19.4 \%$ and $41 \%$ respectively. In our study DMSA scans were required in $42 \%$ patients with ESBL UTI and an abnormality was detected in $76.1 \%$ of them. Similarly, DMSA scan abnormality was found in $23 \%$ of patients with ESBL UTI with higher rates of renal scaring in the study by Dotis $\mathrm{J}$, et $a l^{4}$. In our study, the most common organism isolated in ESBL UTI patients was Escherichia coli (81\%) followed by Klebsiella $(16 \%)$. This was comparable to the study by Hanna-Wakim RH, et al ${ }^{17}$ where Escherichia coli was isolated in $79.4 \%$ of patients and Klebsiella in $7.9 \%$ of patients with ESBL UTI.

In our study when binary logistic regression analysis was done for risk factors between cases and controls as non ESBL UTI, underlying renal problems, antibiotic use within last 3 months, hospitalization within the last 3 months, catheterization within the last 3 months and previous UTI were found to be independent risk factors causing ESBL UTI [Table 5]. In our study, $20 \%, 64 \%$ and $15.3 \%$ of patients with ESBL UTI required hospital stay of less than 4 days, 4 to 7 days and more than 7 days respectively [Figure 1]. Dayan $\mathrm{N}$, et $a l^{20}$ and Fan $\mathrm{N}$, et $a l^{21}$ demonstrated no significant difference in duration of hospital stay in patients with ESBL UTI and in patients with non ESBL UTI. Dotis J, et $a l^{4}$ and Topalogulu R, et $a l^{19}$ 
demonstrated that patients with ESBL UTI required longer duration of hospital stay than with non ESBL UTI. In our study, out of 100 ESBL patients, 70 patients were followed up after treatment for a period of 3 months. Among this $14(20 \%)$ had another episode of UTI [Figure 1]. Fan N, et $a l^{21}$ found no statistical difference in recurrent UTI among patients with ESBL UTI and non ESBL UTI,

There were some limitations. As this is a single centre study conducted at a tertiary care hospital, the demography and socio-economic distribution may be biased. There are probably a few hospital acquired infections in the current study sample despite strict adherence to the study methodology. Even though the urine culture samples were plated at the earliest and in cases of suspicious results they were repeated, there is still a chance of minimal contamination. Therefore a community based multi-centric study is suggested to confirm the findings of this study and reduce the limitations.

\section{Conclusions}

ESBL UTI was common in the age group 2 months to 2 years and was more common in males. Fever was the most common presentation. Underlying renal problems, recent antibiotic use, recent hospitalization, recent catheterization, previous UTI and antibiotic prophylaxis were found to be significant risk factors for ESBL UTI. Higher rates of pyelonephritis, voiding dysfunction, renal abnormalities and VUR were detected in ultrasonogram of patients with ESBL UTI. VUR was more common in patients with ESBL UTI. Renal scarring was more frequent in patients with ESBL UTI. Recurrence of UTI was more likely in patients with ESBL UTI.

\section{References}

1. Awean GZ, Salameh K, Elmohamed H, Alshmayt H, Omer MR. Prevalence of ESBL urinary tract infection in children. Journal of Advanced Pediatrics and Child Health 2019; 2(1): 4-7. https://doi.org/10.29328/journal.japch.100 1004

2. Udani S. Antimicrobial therapy in nosocomial infections. In Shah NK, Singhal $\mathrm{T}$, editors. IAP sub-speciality series on Rational Antimicrobial Practices in Pediatrics (Under IAP Action Plans 2006) 1 st ed. 2006; 216-20.

3. Hellerstein S. Urinary tract infections. Pediatric Clinics of North America 1995; 42: 1433-57.
https://doi.org/10.1016/S00313955(16)400 92-1

4. Dotis J, Printza N, Marneri A, Gidaris D, Papachristou F. Urinary tract infections caused by extended-spectrum betalactamase producing bacteria in children: a matched case-control study. Turkish Journal of Pediatrics 2013; 55: 571-4.

5. Kizilca O, Siraneci R, Yilmaz A, Hatipoglu N, Ozturk E, Kiyak A et al. Risk factors for community-acquired urinary tract infection caused by ESBL-producing bacteria in children. Paediatrics and International Child Health 2012; 54(6): 858-62.

https://doi.org/10.1111/j.1442200X.2012.0 3709.x

6. Camacho-Cruz J, Martinez JM, Cufino JM, Moreno GC, Murillo CR, Fuentes MA, et al. Extended-spectrum $\beta$-lactamaseproducing enterobacteriaceae causing community-acquired urinary tract infections in children in Colombia. Indian Pediatrics 2021; 58(2): 144-8. https://doi.org/10.1007/s13312-021-21318

7. Balasubramanian S, Kuppuswamy D, Padmanabhan S, Chandramohan V, Amperayani S. Extended-spectrum betalactamase-producing community-acquired urinary tract infections in children: Chart review of risk factors. Journal of Global Infectious Diseases 2018; 10(4):222. https://doi.org/10.4103/0974777X.246391

8. Bukhari A, Nunez DS, Etinger V, Clemente M, Gruber J, Lozano MM, et al. 1435. Urinary tract infections due to extended-spectrum beta-lactamaseproducing bacteria in hospitalized children: Demographics, risk factors, clinical characteristics, imaging and treatment. Open Forum Infectious Diseases 2019; 6 (Suppl. 2): S524.

https://doi.org/10.1093/ofid/ofz360.1299

9. Agegnehu A, Worku M, Nigussie D, Lulu B, Tadesse BT. Paediatric febrile urinary tract infection caused by ESBL producing enterobacteriaceae species. BioMed Research International 2020; 2020: Article ID 6679029

https://doi.org/10.1155/2020/6679029 
10. Lukac PJ, Bonomo RA, Logan LK. Extended-spectrum $\quad \beta$-lactamaseproducing enterobacteriaceae in children: old foe, emerging threat. Clinical Infectious Diseases 2015; 60(9): 1389-97. https://doi.org/10.1093/cid/civ020

11. Bagga A, Babu K, Kanitkar M, Srivastava RN. Consensus statement on management of urinary tract infections: Indian pediatric nephrology group Indian academy of pediatrics. Indian Pediatrics 2001; 38(10): 1106-15

12. Mohammed A, Mohammed S, Khan AU. Etiology and antibiotic resistance patterns of community acquired urinary tract infections in JNMC hospital, Aligarh, India. Annals of Clinical Microbiology and Antimicrobials 2007; 6:4-11.

https://doi.org/10.1186/1476-0711-6-4

13. Kaur N, Sharma S, Malhotra S, Madan $P$, Hans C. Urinary tract infection: Etiology and antimicrobial resistance pattern in infants from a tertiary care hospital in Northern India. Journal of Clinical and Diagnostic Research 2014; 8(10): 1-39.

https://doi.org/10.7860/JCDR/2014/8772.4 919

14. Sridhar Rao PN, Basavarajappa KG, Krishna GL. Detection of extended spectrum beta lactamase from clinical isolates in Davangere. Indian Journal of Pathology and Microbiology 2008; 5(4): 4979.

https://doi.org/10.4103/0377-4929.43739

15. Tsering DC, Das S, Adhiakari L, Pal R and Singh TS. Extended spectrum beta lactamase detection in gram negative bacilli of nosocomial origin. Journal of Global Infectious Diseases 2009; 1(2): 8792.

https://doi.org/10.4103/0974-777X.56247

16. Al-Otaibi FE, Bukhari E. Clinical and laboratory profiles of urinary tract infections caused by extended- spectrum beta-lactamase-producing Escherichia coli in a tertiary care center in central Saudi Arabia. Saudi Medical Journal 2013; 34(2):171-6.

17. Hanna-Wakim RH, Ghanem ST, El Helou MW, Sarah A, Rouba A et al. Epidemiology and characteristics of urinary tract infections in children and adolescents. Frontiers in Cellular and Infection Microbiology 2015; 5: 45. https://doi.org/10.3389/fcimb.2015.00045

18. Taneja N, Chatterjee S, Singh M, Singh S, Sharma M. Paediatric urinary tract infections in a tertiary care center from north India. Indian Journal of Medical Research 2010; 131:101-10.

19. Topaloglu R, Er I, Dogan B, Bilginer $\mathrm{Y}$, Ozaltin F, Besbas $\mathrm{N}$, et al. Risk factors in community-acquired urinary tract infections caused by ESBLproducing bacteria in children. Pediatric Nephrology 2010; 25(5): 919-25. https://doi.org/10.1007/s00467-009-14313

20. Dayan N, Dabbah H, Weissman I, et al. Urinary tract infection in CAESBL producing and non-producing bacteria: a comparative study. Journal of Pediatrics 2013; 163(5): 1417-21.

https://doi.org/10.1016/j.jpeds.2013.06.07 8

21. Fan N, Chen H, Chen C, Ou L, Lin T, Tsai et al. Rise of community-onset urinary tract infection caused by extended-spectrum Beta lactamase producing Escherichia coli in children. Journal of Microbiology, Immunology and Infection 2014; 47(5): 399-405. https://doi.org/10.1016/j.jmii.2013.05.006 